\title{
Glycaemic index of common foods tested in the UK and India
}

\author{
C. J. K. Henry ${ }^{1}$, H. J. Lightowler ${ }^{1}$, K. Newens ${ }^{1}$, V. Sudha ${ }^{2}$, G. Radhika ${ }^{2}$, R. M. Sathya ${ }^{2}$ and V. Mohan ${ }^{2}$ \\ ${ }^{1}$ Nutrition and Food Science Group, School of Life Sciences, Oxford Brookes University, Gipsy Lane Campus, Headington, Oxford \\ $O X 3 O B P, U K$ \\ ${ }^{2}$ Madras Diabetes Research Foundation, 4 Conran Smith Road, Gopalapuram, Chennai-600 086, India \\ (Received 23 April 2007 - Revised 8 August 2007 - Accepted 14 August 2007)
}

The aim of this study was to ascertain whether international glycaemic index (GI) values, predominantly developed using peoples living in Europe, North America or Australia, are applicable to Asian Indians resident in their own country. Thirty-four Caucasian subjects were recruited in Oxford, UK and thirteen Asian Indian subjects in Chennai, India. Two types of sweet biscuits and three breakfast cereals were tested for glycaemic response in each group. Subjects were served equivalent available carbohydrate amounts $(50 \mathrm{~g})$ of test foods and a reference food (glucose), on separate occasions. Capillary blood glucose was measured from finger-prick samples in fasted subjects $(-5$ and 0 min) and at $15,30,45$, 60, 90 and $120 \mathrm{~min}$ after starting to eat. For each test food, the incremental area under the curve (IAUC) and GI values were determined. Although the glycaemic response to the reference food was higher in Asian Indian subjects compared with UK Caucasian subjects (IAUC $219 \mathrm{mmol} / \mathrm{min}$ per litre v. $157 \mathrm{mmol} / \mathrm{min}$ per litre, respectively; $P<0 \cdot 01$ ), there was no significant difference in GI values of the five test foods between the two groups. This is the first study known to the authors to examine the role of ethnicity on GI when the subjects are resident in their own countries. The findings from this study have important implications for the use of the GI concept worldwide and support the application of international values to different ethnic groups. The higher glycaemic response to all foods in Asian Indians may represent another mechanism for increased diabetes susceptibility among Asian Indians.

Glycaemic index: Glycaemic response: Ethnicity: Asian Indians

The concept of glycaemic index (GI) was first developed by Jenkins and colleagues over a quarter of a century ago ${ }^{1}$ and has proven to be an important and innovative concept in nutritional science. There is an important body of evidence to support the use and application of low GI diets, not only in the management or prevention of diabetes ${ }^{2-5}$ but also in $\mathrm{CVD}^{6,7}$. In addition, low GI foods have been associated with improved insulin sensitivity ${ }^{8}$ and increased colonic fermentation 9 .

One of the major impediments in the use and application of GI tables worldwide has been the uncertainty of the reliability and applicability of international tables, predominantly developed using peoples living in Europe, North America or Australia, to different ethnic groups. An early study found that rural African subjects and Caucasian subjects had similar GI values for the same foods ${ }^{10}$. Similarly, in Australia, the GI values of Vietnamese foods tested in Asian and Caucasian subjects did not differ ${ }^{11}$. However, most of the previous studies examined the role of ethnicity on GI when both subjects were resident in Europe, South Africa or Australia.

The GI concept may be of particular benefit in India due to the high prevalence of diabetes and impaired glucose tolerance. The National Urban Diabetes Survey found that $12 \%$ of city-dwelling Indians have type 2 diabetes and $13 \%$ of those under 40 years of age have impaired glucose tolerance ${ }^{12}$.
Moreover, the prevalence of diabetes in urban India continues to rise. The recent Chennai Urban Rural Epidemiology Study reports that the prevalence of diabetes has reached $14.3 \%{ }^{13}$. In particular, Asian Indians are more insulin resistant than other ethnic groups ${ }^{14}$. A diet-based intervention and consideration of GI in dietary therapy for diabetics in India may be a more appropriate and financially suitable strategy than one based on pharmaceutical drugs.

In light of the above, the aim of the present study was to compare the GI values of the same foods tested in Caucasians living in the UK and Asian Indians living in India. This is the first study known to the authors to examine the role of ethnicity on GI when the subjects are resident in their own countries. The results obtained will enable us to determine whether the suitability of GI values predominantly obtained from Western societies, notably Europe, North America and Australia, may be successfully applied to other regions of the world, in this case Asia.

\section{Experimental methods}

Subjects

Thirty-four Caucasian (fourteen male, twenty female) and thirteen Asian Indian (seven male, six female) healthy subjects 
were recruited to take part in the present study at Oxford Brookes University, UK and the Madras Diabetes Research Foundation, Chennai, India, respectively. Subjects were randomly selected from a pool currently participating in studies on the glycaemic response of different foods. Exclusion criteria were as follows: age $<18$ or $>65$ years; BMI $>30 \mathrm{~kg} / \mathrm{m}^{2}$; fasting blood glucose value $>6.1 \mathrm{mmol} / \mathrm{l}$. Ethical approval for the study was obtained from the University Research Ethics Committee at Oxford Brookes University and the Institutional Ethics Committee of the Madras Diabetes Research Foundation. Subjects were given full details of the study protocol and the opportunity to ask questions. All subjects gave written informed consent prior to participation.

Characteristics of the subjects are shown in Table 1. All measurements were made in the fasting state. Height was recorded to the nearest $\mathrm{cm}$ using a stadiometer (Seca Ltd, Birmingham, UK), with subjects standing erect and without shoes. Body weight (recorded to the nearest $0.1 \mathrm{~kg}$ ) and body fat were measured using the Tanita BC-418 MA (Tanita UK Ltd, Yiewsley, UK), with subjects wearing light clothing and no shoes. BMI was calculated using the standard formula: weight $(\mathrm{kg}) /$ height $^{2}\left(\mathrm{~m}^{2}\right)$. Waist and hip circumference were measured with a standard tape measure at the midpoint between the coastal margins of the ribs and the upper margin of iliac crest and at the level of the greater trochanters on both sides, respectively. Blood pressure was measured with an automatic device (A\&D Company Ltd, Tokyo, Japan) in UK Caucasian subjects and the Omron electronic machine (OMRON Corporation, Tokyo, Japan) in Asian Indian subjects.

\section{Study protocol}

The following protocol was adhered to in both the UK and in India. The protocol used to measure GI was adapted from that described by Wolever et al. ${ }^{15}$ and is in line with procedures recommended by the FAO/WHO ${ }^{16}$. The FAO/WHO state that to determine the GI of a food, tests should be repeated in six or more subjects; moreover, it has been suggested that testing in ten subjects provides a degree of power and precision ${ }^{17}$. Thus, for each test food, ten subjects (selected from the subjects

Table 1. Subject characteristics*

(Mean values and standard deviations)

\begin{tabular}{|c|c|c|c|c|c|}
\hline & \multicolumn{2}{|c|}{$\begin{array}{l}\text { UK Caucasian } \\
\qquad(n 34)\end{array}$} & \multicolumn{2}{|c|}{$\begin{array}{l}\text { Asian Indian } \\
\quad(n 13)\end{array}$} & \multirow[b]{2}{*}{$P$ value } \\
\hline & Mean & SD & Mean & SD & \\
\hline Age (years) & 37 & 13 & $24 \cdot 8$ & 3 & $<0.001$ \\
\hline Height (m) & $1 \cdot 70$ & 0.09 & 1.66 & $0 \cdot 10$ & 0.109 \\
\hline Weight (kg) & $71 \cdot 1$ & $12 \cdot 0$ & $60 \cdot 7$ & $9 \cdot 4$ & 0.008 \\
\hline BMI $\left(\mathrm{kg} / \mathrm{m}^{2}\right)$ & $24 \cdot 4$ & 3.4 & $22 \cdot 1$ & $1 \cdot 3$ & 0.001 \\
\hline Body fat (\%) & $26 \cdot 5$ & $11 \cdot 0$ & $24 \cdot 1$ & 8.5 & 0.482 \\
\hline $\begin{array}{l}\text { Waist circumference } \\
(\mathrm{cm})\end{array}$ & $82 \cdot 5$ & $11 \cdot 0$ & $79 \cdot 2$ & 5.9 & 0.202 \\
\hline \multicolumn{6}{|l|}{ Blood pressure } \\
\hline Systolic $(\mathrm{mmHg})$ & 121 & 20 & 112 & 14 & 0.176 \\
\hline Diastolic $(\mathrm{mmHg})$ & 80 & 10 & 72 & 11 & 0.040 \\
\hline $\begin{array}{l}\text { Fasting blood glucose } \\
(\mathrm{mmol} / \mathrm{l})\end{array}$ & $4 \cdot 7$ & 0.4 & $4 \cdot 7$ & 0.5 & 0.694 \\
\hline
\end{tabular}

${ }^{*}$ For details of subjects and procedures, see Experimental methods. recruited to the present study) were tested. On the day prior to a test, subjects were asked to restrict their intake of alcohol and caffeine-containing drinks and to restrict their participation in intense physical activity (e.g. long periods at the gym, excessive swimming, running, aerobics). Subjects were also told not to eat or drink after 21.00 hours the night before a test, although water was allowed in moderation.

\section{Test foods}

Five foods were tested in both centres, two sweet biscuits and three breakfast cereals. The choice of foods was based on nonperishable foods and foods that were easily acceptable by the Asian Indian consumers. All foods were tested in $50 \mathrm{~g}$ available carbohydrate amounts and compared with a reference food (glucose). Available carbohydrate values were provided by the manufacturers. All products were consumed dry with the exception of the cereal biscuits, which were served with semi-skimmed milk according to the manufacturer's instructions.

In accordance with $\mathrm{FAO} / \mathrm{WHO}$ recommendations ${ }^{16}$, subjects tested each test food once and the reference food three times in random order on separate days, with at least $1 \mathrm{~d}$ gap between measurements to minimise carry-over effects. Subjects were studied in the morning after a $12 \mathrm{~h}$ overnight fast. Subjects consumed the reference/test food at a comfortable pace, within $15 \mathrm{~min}$. The test foods and reference food were served with $200 \mathrm{ml}$ water; further $200 \mathrm{ml}$ water was given during the subsequent $2 \mathrm{~h}$. Subjects were encouraged to keep physical activity to a minimum during the testing.

\section{Blood glucose measurements}

The testing procedure was followed exactly in both centres. Fasting blood samples were taken at $-5 \mathrm{~min}$ and $0 \mathrm{~min}$ before consumption of the food and the baseline value taken as a mean of these two values. The reference/test food was consumed immediately after this and further blood samples were taken at 15, 30, 45, 60, 90 and 120 min after starting to eat. Blood was obtained by finger-prick using Unistik ${ }^{\circledR} 3$ single-use lancing device (Owen Mumford, Woodstock, UK). Recent reports suggest that capillary rather than venous blood sampling is preferred for reliable GI testing $^{16-18}$. Prior to a finger-prick, subjects were encouraged to warm their hand to increase blood flow. Fingers were not squeezed to extract blood from the fingertip in order to minimize plasma dilution. Blood glucose was measured using automatic analysers (Ascensia Contour ${ }^{\circledR}$; Bayer HealthCare, Newbury, UK), which were calibrated daily using control solutions from the manufacturer and also regularly calibrated against a clinical dry chemistry analyser (Reflotron ${ }^{\circledR}$ Plus; Roche, Welwyn Garden City, UK) and the HemoCue Glucose $201+$ analyser (HemoCue ${ }^{\circledR}$ Ltd, Dronfield, UK). Previous work has shown a very strong correlation and good agreement between blood glucose measurements using the automatic analyser and the HemoCue analyser ${ }^{19}$.

\section{Calculation of glycaemic index}

For the reference food and each test food, the incremental area under the curve (IAUC), ignoring the area beneath the 
baseline, was calculated geometrically ${ }^{16}$. The mean, standard deviation and CV of the IAUC of each subject's repeated reference food were calculated. The IAUC for each test food eaten by each subject was expressed as a percentage of the mean IAUC for the reference food eaten by the same subject. The GI of each test food was taken as the mean for the whole group.

\section{Statistical analysis}

Statistical analysis was performed using the Statistical Product and Service Solutions software (SPSS version 11.0.1; Chicago, IL, USA). Data are presented as means, standard deviations and standard errors. Prior to statistical analysis, the normality of the data was tested using the Shapiro-Wilks statistic. Levels of inter- and intra-individual variation of the three reference (glucose) tests were assessed by determining $\mathrm{CV} \%$. Differences in physical characteristics, IAUC values and GI values between the two study groups were assessed using independent samples $t$ tests or Mann-Whitney tests where appropriate (GI values for sweetmeal biscuits; IAUC values for sweetmeal biscuits, sweet biscuits and malted wholegrain cereal). Using analysis of covariance, the effects of age, BMI and blood pressure in GI were analysed for each of the five foods tested. There was no significant effect of any of these covariates on the GI of the foods. Statistical significance was set at $P<0 \cdot 05$.

\section{Results}

Table 1 compares the characteristics of the UK Caucasian and Asian Indian subjects. Mean fasting blood glucose value of the two groups was very similar; however, there were significant differences in other characteristics. The BMI of the Asian Indian subjects was significantly lower than the UK Caucasians $(P=0.001)$, despite similar mean body fat percentage.

Mean IAUC of the reference food was significantly higher in Asian Indian subjects (219 (SD 75) $\mathrm{mmol} / \mathrm{min}$ per litre) compared with UK Caucasian subjects (157 (SD 63) mmol/ min per litre; $P=0 \cdot 007$ ). However, the variability of glycaemic response to the reference tests was similar between the two groups. Mean intra-individual variation in glycaemic response to the three reference tests was $22 \% \mathrm{CV}$ for UK Caucasian subjects and $21 \% \mathrm{CV}$ for Asian Indian subjects $(P=0 \cdot 780)$. These values are consistent with previously reported data in normal subjects ${ }^{20}$. The inter-individual variation in glycaemic response to the reference tests was $41 \%$ $\mathrm{CV}$ and $34 \% \mathrm{CV}$ for the UK Caucasians and Asian Indians, respectively.

Figs. 1-5 show the incremental glycaemic response curves for the five test foods in the UK Caucasians and Asian Indians. Although the shape of the incremental glycaemic response curves appears similar between the UK Caucasian and Asian Indian subjects, the IAUC values for all five test foods were higher in the Asian Indian group (Table 2). In particular, compared with the Asian Indian subjects, IAUC was significantly lower for sweet biscuits $(P=0.009)$ and malted wheat cereal $(P=0.032)$.

Nevertheless, in the calculation of GI, the absolute IAUC values for each food are expressed as a percentage of the mean IAUC for the reference food. As shown in Table 3, when the GI values were calculated in each group, these did not differ significantly in all five foods tested.

\section{Discussion}

This is the first study known to the authors to examine the role of ethnicity on GI when the subjects are resident in their own countries. The importance of ethnicity was to establish whether feeding identical foods in two regions of the world would elicit similar or dissimilar GI values. The present study has shown that although the glycaemic response to the foods was higher in Asian Indian subjects compared with UK Caucasian subjects, there were no significant differences in the GI values of the same foods between the two groups. Moreover, differences in GI values of the foods between the two groups (ranging from nil (cereal biscuit) to 16 (sweet biscuits) GI points) were below the threshold of 18 points, as suggested by Wolever et $a .^{18}$. The results obtained will enable us to propose the suitability of GI values predominantly obtained from Western societies, notably Europe, North (a)

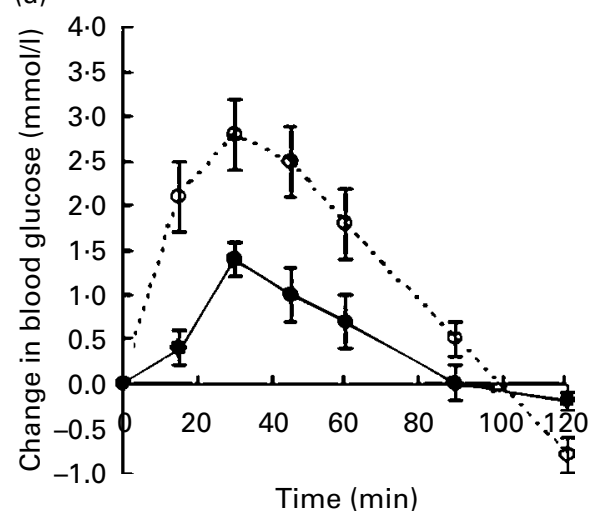

(b)

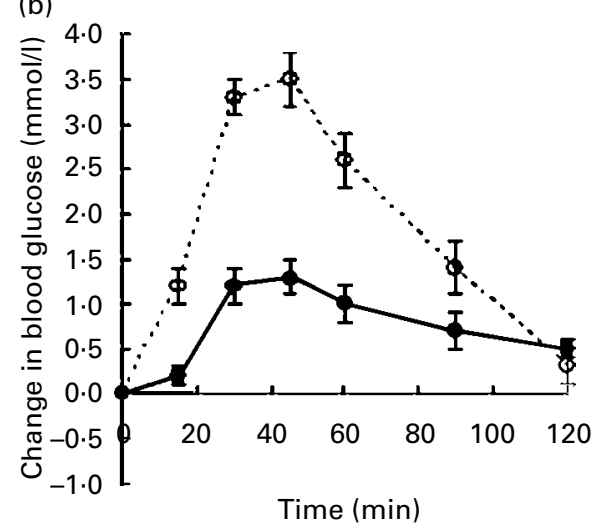

Fig. 1. Incremental area under the curve for reference food $(\bigcirc)$ and sweetmeal biscuit $(\bullet)$ for (a) UK Caucasian and (b) Asian Indian subjects. Each point represents the average change in blood glucose for ten subjects. Standard errors of the mean values are represented by vertical bars. For details of subjects and procedures, see Experimental methods. 

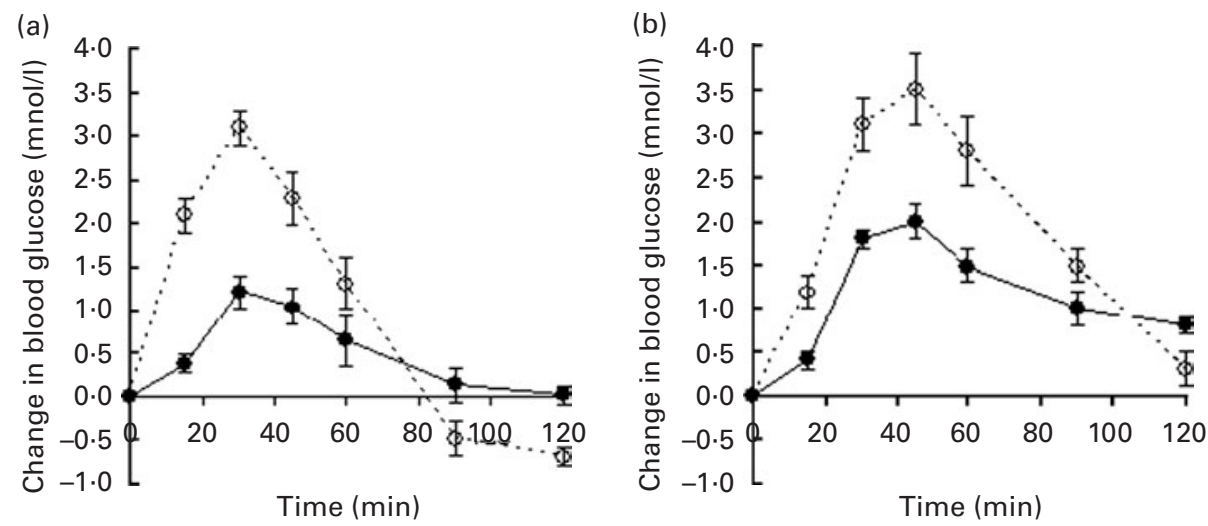

Fig. 2. Incremental area under the curve for sweet biscuit for (a) UK Caucasian and (b) Asian Indian subjects. Each point represents the average change in blood glucose for ten subjects. Standard errors of the mean values are represented by vertical bars. For details of subjects and procedures, see Experimental methods.

(a)

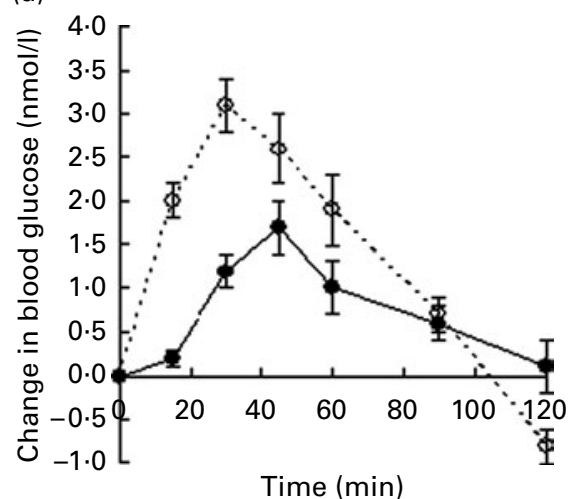

(b)

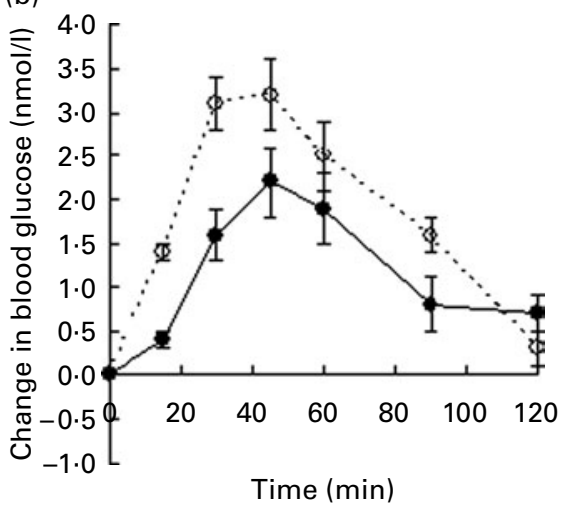

Fig. 3. Incremental area under the curve for malted wholewheat cereal for (a) UK Caucasian and (b) Asian Indian subjects. Each point represents the average change in blood glucose for ten subjects. Standard errors of the mean values are represented by vertical bars. For details of subjects and procedures, see Experimental methods.

(a)

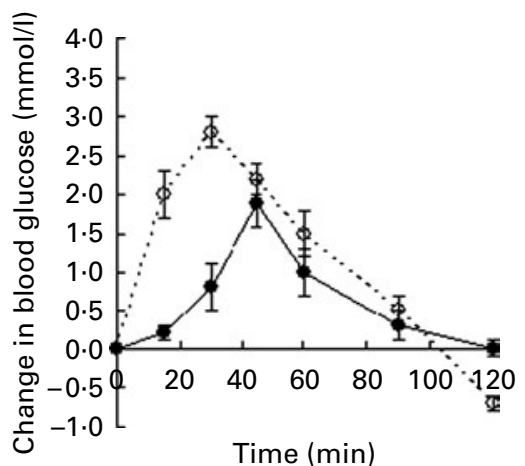

(b)

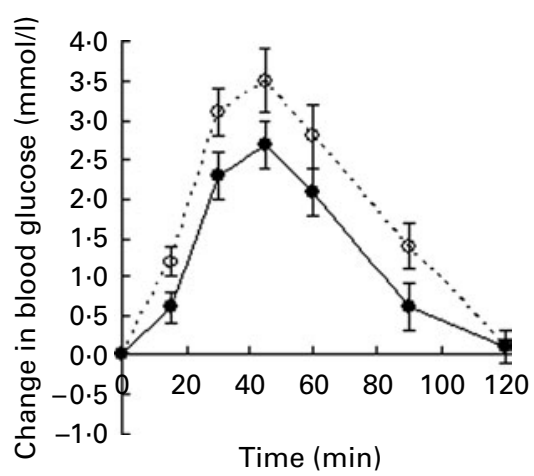

Fig. 4. Incremental area under the curve for malted wheat cereal for (a) UK Caucasian and (b) Asian Indian subjects. Each point represents the average change in blood glucose for ten subjects. Standard errors of the mean values are represented by vertical bars. For details of subjects and procedures, see Experimental methods.

America and Australia, to other regions of the world, in this case Asia.

The glucose curves of the Asian Indian subjects were similar to those of the UK Caucasian subjects, although the change from baseline was greater indicating a higher glycaemic response. The IAUC for the reference food was also higher in the Asian Indian subjects. Despite the higher glycaemic response to the reference food and test foods, the fasting blood glucose of the two ethnic groups was very similar. The persistently higher glycaemic response to all foods observed in Asian Indian subjects may be ascribed to higher glucose intolerance rates. Our finding of a greater glycaemic 


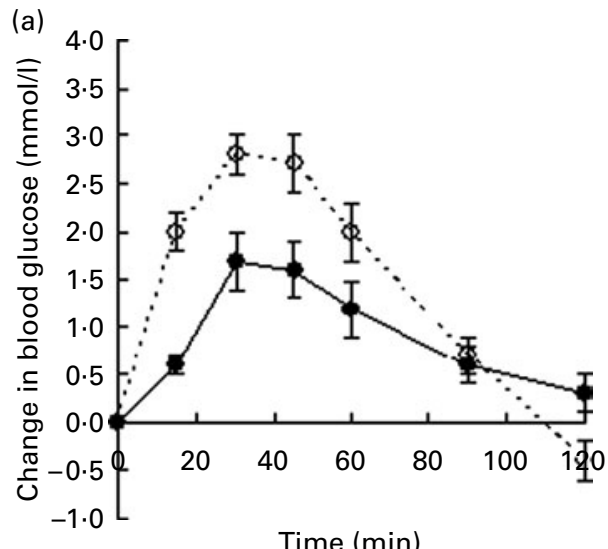

Time $(\min )$

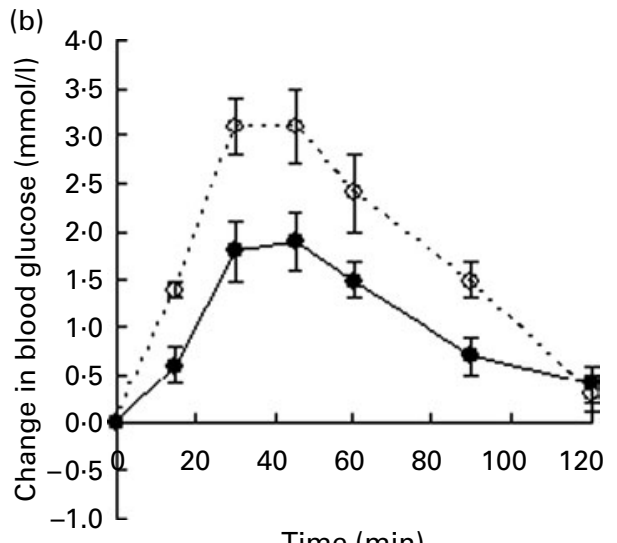

Time $(\min )$

Fig. 5. Incremental area under the curve for cereal biscuit for (a) UK Caucasian and (b) Asian Indian subjects. Each point represents the average change in blood glucose for ten subjects. Standard errors of the mean values are represented by vertical bars. For details of subjects and procedures, see Experimental methods.

Table 2. Incremental area under the curve values ( $\mathrm{mmol} / \mathrm{min}$ per litre) $\dagger$ (Mean values with their standard errors of the mean)

\begin{tabular}{lrrrrrrr}
\hline & \multicolumn{2}{c}{ UK Caucasian } & & \multicolumn{2}{c}{ Asian Indian } & \\
\cline { 2 - 3 } & Mean & SEM & & Mean & SEM & P value \\
\hline Sweetmeal biscuit & 66 & 14 & & 94 & 13 & 0.063 \\
Sweet biscuit & 92 & 17 & & 140 & 12 & 0.009 \\
Malted wholewheat cereal & 93 & 16 & & 141 & 26 & 0.113 \\
Malted wheat cereal & 94 & 13 & & 153 & 22 & 0.032 \\
Cereal biscuit* & 111 & 18 & & 125 & 15 & 0.546 \\
\hline
\end{tabular}

* Prepared with $243 \mathrm{ml}$ semi-skimmed milk.

†For details of subjects and procedures, see Experimental methods.

response in the Asian Indian group may not be unexpected, particularly in an urban area such as Chennai, where glucose intolerance rates are high ${ }^{12}$. In the present study, all Asian Indian subjects were healthy volunteers. Thus, this may be another aspect of the 'Asian Indian Phenotype'21.

Brouns et al. ${ }^{17}$ concluded that in order to reduce intra-subject variation, subjects should be healthy and not suffer from diabetes. For this reason, it is important to consider the effect of the higher glycaemic response on intra-subject variation as measured by the $\mathrm{CV}$ of the reference test foods. In fact, the $\mathrm{CV}$ of the Indian subjects was almost identical to the CV of the UK subjects ( $21 \% v .22 \%)$ and consistent with previously reported values ${ }^{20}$. This is a very encouraging finding and promotes the application of GI values worldwide.

The current study supports the findings of previous research. Walker \& Walker ${ }^{10}$ found that rural African subjects and Caucasian subjects had similar GI values for the same foods. In Australia, the GI of Vietnamese foods tested in Asian and Caucasian subjects did not differ ${ }^{11}$. The present study adds weight to the belief that GI is not affected by the ethnicity of the subjects, even in populations at risk from diabetes in their native country. An additional unique aspect of this study was the minimisation of inter-laboratory differences by replication of the test foods, protocol and equipment.

In conclusion, the findings from the present study have important implications for the use of the GI concept worldwide. Despite the Western origin of international GI tables, this study supports the application of international values to different ethnic groups and promotes the use of international GI tables within the Asian Indian context. The higher glycaemic response to all foods in Asian Indians may represent another mechanism for increased diabetes susceptibility among Asian Indians. Moreover, in a country of high diabetes prevalence, consideration of GI dietary therapy is likely to be beneficial to help curb the rising incidence of this disease, as well as many other chronic diseases such as CVD.

Table 3. Glycaemic index (Gl) values and classificationt (Mean values with their standard errors of the mean)

\begin{tabular}{|c|c|c|c|c|c|c|c|}
\hline & \multicolumn{3}{|c|}{ UK Caucasian } & \multicolumn{3}{|c|}{ Asian Indian } & \multirow[b]{3}{*}{$P$ value } \\
\hline & \multicolumn{2}{|c|}{ Gl value } & \multirow[b]{2}{*}{ Gl classification } & \multicolumn{2}{|c|}{ Gl value } & \multirow[b]{2}{*}{ Gl classification } & \\
\hline & Mean & SEM & & Mean & SEM & & \\
\hline Sweetmeal biscuit & 39 & 4 & Low & 42 & 4 & Low & 0.436 \\
\hline Sweet biscuit & 47 & 6 & Low & 63 & 4 & Medium & 0.052 \\
\hline Malted wholewheat cereal & 56 & 5 & Medium & 60 & 7 & Medium & 0.465 \\
\hline Malted wheat cereal & 60 & 5 & Medium & 68 & 5 & Medium & 0.259 \\
\hline Cereal biscuit* & 59 & 7 & Medium & 59 & 5 & Medium & 0.957 \\
\hline
\end{tabular}

* Prepared with $243 \mathrm{ml}$ semi-skimmed milk.

†For details of subjects and procedures, see Experimental methods. 


\section{References}

1. Jenkins DJA, Wolever TMS, Taylor RH, Barker H, Fielden H, Baldwin JM, Bowling AC, Newman HC, Jenkins AL \& Goff DV (1981) Glycemic index of foods: a physiological basis for carbohydrate exchange. Am J Clin Nutr 34, 362-366.

2. Brand JC, Colagiuri S, Crossman S, Allen A, Roberts DCK \& Truswell AS (1991) Low-glycemic index foods improve longterm glycemic control in NIDDM. Diabetes Care 14, 95-101.

3. Frost G, Wilding J \& Beecham J (1994) Dietary advice based on the glycaemic index improves dietary profile and metabolic control in Type 2 diabetic patients. Diabet Med 11, 397-401.

4. Salmeron J, Ascherio A, Rimm EB, Colditz GA, Spiegelman D, Jenkins DJ, Stampfer MJ, Wing AL \& Willet EC (1997) Dietary fiber, glycaemic load, and risk of NIDDM in men. Diabetes Care 20, 545-550.

5. Salmeron J, Manson JE, Stampfer MJ, Colditz GA, Wing AL \& Willet EC (1997) Dietary fiber, glycaemic load, and risk of noninsulin-dependent diabetes mellitus in women. JAMA $\mathbf{2 7 7}$, $472-477$.

6. Jenkins DJA, Wolever TMS, Kalmusky J, et al. (1987) Lowglycemic index diet in hyperlipidemia: use of traditional starchy foods. Am J Clin Nutr 46, 66-71.

7. Frost G, Leeds AA, Dore CJ, Madeiros S, Brading S \& Dornhorst A (1999) Glycaemic index as a determinant of serum HDL-cholesterol concentration. Lancet 353, 1045-1048.

8. Frost G, Leeds A, Trew G, Margara R \& Dornhorst A (1998) Insulin sensitivity in women at risk of coronary heart disease and the effect of a low glycemic diet. Metabolism 47, 1245-1251.

9. Jenkins DJA, Wolever TMS, Collier GR, Ocana A, Rao AV, Buckley G, Lam Y, Mayer A \& Thompson LU (1987) Metabolic effects of a low glycemic index diet. Am J Clin Nutr 46, 968-975.

10. Walker AR \& Walker BF (1984) Glycaemic index of South African foods determined in rural blacks - a population at low risk of diabetes. Hum Nutr Clin Nutr 38, 215-222.
11. Chan HM, Brand-Miller JC, Holt SH, Wilson D, Rozman M \& Petocz P (2001) The glycaemic index values of Vietnamese foods. Eur J Clin Nutr 55, 1076-1083.

12. Ramachandran A, Snehalatha C, Kapur A, Vijay V, Mohan V, Das AK, Rao PV, Yajnik CS, Prasanna Kumar KM \& Nair JD (2001) High prevalence of diabetes and impaired glucose tolerance in India: National Urban Diabetes Survey. Diabetologia 44, 1094-1101.

13. Mohan V, Deepa M, Deepa R, Shanthirani CS, Farooq S, Ganesan A \& Datta M (2006) Secular trends in the prevalence of diabetes and impaired glucose tolerance in urban South India - the Chennai Urban Rural Epidemiology Study (CURES-17). Diabetologia 49, $1175-1178$

14. Abate N \& Chandalia M (2003) The impact of ethnicity on type 2 diabetes. J Diabetes Complications 17, 39-58.

15. Wolever TMS, Jenkins DJ, Jenkins AL \& Josse RG (1991) The glycemic index: methodology and clinical implications. Am J Clin Nutr 54, 846-854.

16. Food and Agriculture Organization/World Health Organization (1998) Carbohydrates in Human Nutrition. Report of a Joint FAO/WHO Expert Consultation. Rome: FAO.

17. Brouns F, Bjorck I, Frayn KN, Gibbs AL, Lang V, Slama G \& Wolever TMS (2005) Glycaemic index methodology. Nutrition Research Reviews 18, 145-171.

18. Wolever TMS, Vorster HH, Bjorck I, et al. (2003) Determination of the glycaemic index of foods: interlaboratory study. Eur J Clin Nutr 57, 475-482.

19. Henry CJK, Lightowler HJ, Strik CM, Renton R \& Hails S (2005) Glycaemic index and glycaemic load values of commercially available products in the United Kingdom. Br J Nutr 94, 922-930.

20. Wolever TMS (2006) The Glycaemic Index. A Physiological Classification of Dietary Carbohydrate. Wallingford: CABI.

21. Mohan V \& Deepa R (2006) Adipocytokines and the expanding 'Asian Indian Phenotype'. J Assoc Physicians India 54, $685-686$. 